Kansas State University Libraries

New Prairie Press

\title{
ESTIMATING INTRACLASS CORRELATION: OPTIMAL RESULTS USING LIMITED RESOURCES
}

Brent D. Burch

Ian R. Harris

Follow this and additional works at: https://newprairiepress.org/agstatconference

Part of the Agriculture Commons, and the Applied Statistics Commons

\section{(c) (1) $\Theta($}

This work is licensed under a Creative Commons Attribution-Noncommercial-No Derivative Works 4.0 License.

\section{Recommended Citation}

Burch, Brent D. and Harris, Ian R. (2002). "ESTIMATING INTRACLASS CORRELATION: OPTIMAL RESULTS USING LIMITED RESOURCES," Conference on Applied Statistics in Agriculture. https://doi.org/10.4148/ 2475-7772.1199

This is brought to you for free and open access by the Conferences at New Prairie Press. It has been accepted for inclusion in Conference on Applied Statistics in Agriculture by an authorized administrator of New Prairie Press. For more information, please contact cads@k-state.edu. 


\title{
ESTIMATING INTRACLASS CORRELATION: OPTIMAL RESULTS USING LIMITED RESOURCES
}

\author{
Brent D. Burch ${ }^{1}$ and Ian R. Harris ${ }^{2}$ \\ ${ }^{1}$ Department of Mathematics and Statistics \\ Northern Arizona University, Flagstaff, Arizona 86011, U.S.A. \\ ${ }^{2}$ Department of Statistical Science \\ Southern Methodist University, Dallas, Texas 75275, U.S.A.
}

\begin{abstract}
From plant and animal breeding studies to industrial applications, the intraclass correlation coefficient $(\rho)$ is used to measure the proportion of the total variation in the responses that may be attributed to a particular source. Confidence intervals for $\rho$ are used to determine the optimal allocation of experimental material in one-way random effects models. Assuming the sample size is fixed, the authors investigate the number of groups and the number of observations per group required to minimize the expected length of confidence intervals. Examples are used to illustrate the selection of the best design. Both asymptotic and exact results suggest that practitioners should allocate no more than four experimental units per group.
\end{abstract}

\section{Introduction}

One-way random effects models are a collection of designs that have a wide range of applicability. For example, an apple grower may be interested in estimating how much of the variability in the weights of apples is due to the variation of trees in an apple orchard. In an industrial application (see Vangel, 1992) where a product is manufactured in batches, this model serves as a tool to highlight how the batch variability influences the variability in the finished goods. In one-way random effects models the variance components, which we denote by $\sigma_{1}^{2}$ and $\sigma_{2}^{2}$, are the variances of two sources that contribute to the variation of the responses. If $\sigma_{1}^{2}$ is the variance due to trees in the apple orchard, $\sigma_{2}^{2}$ is the variance of the apples within a tree, and all the effects combine linearly, the intraclass correlation coefficient is $\rho=\sigma_{1}^{2} /\left(\sigma_{1}^{2}+\sigma_{2}^{2}\right)$.

In many instances the practitioner would like to accurately estimate $\rho$ while being constrained by a limited sample size. When resources are restricted, the investigator must judiciously select the number of groups and/or the number of measurements per group. In other words, for a given sample size, the investigator wants the best one-way random effects design to estimate $\rho$. See Burch and Harris (2002) for a review of literature related to this topic.

Unlike previous literature, we consider confidence intervals for $\rho$ as a measure of the quality of the design. For equal-tailed intervals having a fixed level of confidence, short intervals are desirable as they indicate with a high degree of certainty plausible values of 
$\rho$. We investigate the optimal allocation of resources in terms of number of groups and number of observations per group to minimize the expected length of confidence intervals for $\rho$. In the apple orchard example, this equates to the following scenario: Suppose 100 apples can be picked from trees in an apple orchard. How many trees and how many apples per tree should be picked to obtain a short confidence interval for $\rho$ ?

The paper is organized as follows. Section 2 provides background information and introduces the notation used in the one-way random effects model. In Section 3, confidence intervals for $\rho$ and the computational aspects associated with the expected length of confidence intervals for $\rho$ are examined. Section 4 gives examples to illustrate the optimal allocation procedure described in this paper. Optimal designs are based on asymptotic and exact computations of the expected length of confidence intervals for $\rho$. Section 5 is discussion and summary.

\section{The One-Way Random Effects Model}

Consider the one-way random effects model given by

$$
Y_{i j}=\mu+A_{i}+e_{i j}
$$

where $i=1, \ldots, a, j=1, \ldots, b_{i}$, and $\sum_{i=1}^{a} b_{i}=n . Y_{i j}$ is the $j^{\text {th }}$ observation associated with the $i^{\text {th }}$ class (or group) of factor $A$. The $a$ groups of $A$ in the model are assumed to be randomly selected from some large population of groups. Furthermore, a random sample of size $b_{i}$ has been obtained from the $i^{\text {th }}$ group. $e_{i j}$ is often referred to as random error. It is assumed that $A_{i} \stackrel{i i d}{\sim} N\left(0, \sigma_{1}^{2}\right), e_{i j} \stackrel{i i d}{\sim} N\left(0, \sigma_{2}^{2}\right)$, and that $A_{i}$ and $e_{i j}$ are mutually independent. In addition, $\sigma_{1}^{2} \geq 0$ and $\sigma_{2}^{2}>0 . \mu$ is a fixed but unknown quantity that represents the overall mean of $Y_{i j}$.

Since $\operatorname{Var}\left(A_{i}\right)=\sigma_{1}^{2}$ and $\operatorname{Var}\left(Y_{i j}\right)=\operatorname{Var}\left(A_{i}\right)+\operatorname{Var}\left(e_{i j}\right)=\sigma_{1}^{2}+\sigma_{2}^{2}$, the parameter $\rho=\sigma_{1}^{2} /\left(\sigma_{1}^{2}+\sigma_{2}^{2}\right)$ is the proportion of the variation in the $Y_{i j}$ 's attributed to factor A. Also note that observations within the same group are correlated since $\operatorname{Cov}\left(Y_{i j}, Y_{i j^{\prime}}\right)=$ $\sigma_{1}^{2}$, and observations from different groups are uncorrelated. In this manner $\rho$ may also be interpreted as the correlation between two observations within the same group. By definition, $0 \leq \rho<1$. In the apple orchard example, if $Y_{i j}$ is the weight of the $j^{\text {th }}$ apple selected from the $i^{\text {th }}$ tree, $\sigma_{1}^{2}$ is the variation due to trees in the orchard, and $\sigma_{2}^{2}$ is the variation due to apples on a tree, then $\rho$ is the proportion of the total variation in apple weights accounted for by the trees. $\rho$ is also the correlation between weights of apples from the same tree.

Since $\rho$ is a function of variance components, and the objective is to select the design which provides the most information about $\rho$, we begin by examining the properties of a set of quadratic forms used to estimate the variance components. The number of quadratic forms and their corresponding distributions depend on the underlying model structure. Using matrix notation, model (1) becomes

$$
\mathbf{Y}=1 \mu+\mathrm{ZA}+\mathrm{e}
$$


where $\mathbf{Y}$ is a $n \times 1$ vector of responses, $\mathbf{1}$ is a $n \times 1$ vector of ones, $\mathbf{A}$ and $\mathbf{e}$ are random vectors of length $a$ and $n$, respectively, and

$$
\mathbf{Z}=\left[\begin{array}{cccc}
\mathbf{1}_{b_{1}} & & & \\
& \mathbf{1}_{b_{2}} & & \\
& & \ddots & \\
& & & \mathbf{1}_{b_{a}}
\end{array}\right]_{n \times a}
$$

where $1_{b_{i}}$ is a $b_{i} \times 1$ vector of ones.

The quadratic forms may be obtained by diagonalizing the variance-covariance matrix of $\mathbf{H}^{\prime} \mathbf{Y}$, a linear transformation of the observations, where $\mathbf{H}$ is a $n \times(n-1)$ matrix whose columns span the space orthogonal to the space spanned by the column of ones and satisfies $\mathbf{H}^{\prime} \mathbf{H}=\mathbf{I}_{\mathbf{n}-\mathbf{1}}$. Note that $\mathbf{H}^{\prime} \mathbf{Y} \sim N\left(\mathbf{0}, \sigma_{2}^{2} \mathbf{I}_{\mathbf{n}-\mathbf{1}}+\sigma_{1}^{2} \mathbf{H}^{\prime} \mathbf{Z} \mathbf{Z}^{\prime} \mathbf{H}\right)$ is a $n-1$ dimensional vector whose distribution does not depend on $\mu$. Let $0 \leq \Delta_{1}<\ldots<\Delta_{d}$ be the distinct eigenvalues of $\mathbf{H}^{\prime} \mathbf{Z} \mathbf{Z}^{\prime} \mathbf{H}$ having multiplicities $r_{1}, \ldots, r_{d}$, respectively. There are at most $a$ unique eigenvalues. There exists an $(n-1) \times(n-1)$ orthogonal matrix $\mathbf{P}$ such that $\mathbf{P}^{\prime}\left(\mathbf{H}^{\prime} \mathbf{Z} \mathbf{Z}^{\prime} \mathbf{H}\right) \mathbf{P}=\operatorname{Diag}\left(\Delta_{1}, \ldots, \Delta_{1}, \ldots, \Delta_{d}, \ldots, \Delta_{d}\right)=\mathbf{D}$ where each $\Delta_{m}$ is repeated $r_{m}$ times, $m=1, \ldots, d$. Then $\mathbf{P}^{\prime} \mathbf{H}^{\prime} \mathbf{Y} \sim N\left(\mathbf{0}, \sigma_{2}^{2} \mathbf{I}_{\mathbf{n}-\mathbf{1}}+\sigma_{1}^{2} \mathbf{D}\right)$ and $\mathbf{P}_{\mathbf{m}}^{\prime} \mathbf{H}^{\prime} \mathbf{Y} \sim N\left(\mathbf{0},\left(\sigma_{2}^{2}+\sigma_{1}^{2} \Delta_{m}\right) \mathbf{I}_{r_{m}}\right)$, $m=1, \ldots, d$ where $\mathbf{P}=\left[\mathbf{P}_{1}, \ldots, \mathbf{P}_{d}\right]$ and each matrix $\mathbf{P}_{m}$ corresponding to $\Delta_{m}$ is of size $(n-1) \times r_{m}$. It follows that $Q_{m}=\mathbf{Y}^{\prime}\left(\mathbf{H P}_{\mathbf{m}} \mathbf{P}_{\mathbf{m}}^{\prime} \mathbf{H}^{\prime}\right) \mathbf{Y}$ and

$$
Q_{m} \sim \frac{\sigma_{2}^{2}}{1-\rho}\left(1+\rho\left(\Delta_{m}-1\right)\right) \chi^{2}\left(r_{m}\right)
$$

where $m=1, \ldots, d$. By construction, $Q_{1}, \ldots, Q_{d}$ are independent. A complete description of the distributional theory associated with the quadratic forms in a one-way random effects model is described by LaMotte (1976). Burch and Iyer (1997) discuss the theory used to construct the quadratic forms and associated eigenvalues in a more general setting.

The total variation in the observations, given by $\sum_{i=1}^{a} \sum_{j=1}^{b_{i}}\left(Y_{i j}-\bar{Y}_{. .}\right)^{2}$ where $\bar{Y}_{\text {.. }}$ is the overall sample mean, may be partitioned (see LaMotte, 1976) as

$$
\begin{aligned}
\sum_{i=1}^{a} \sum_{j=1}^{b_{i}}\left(Y_{i j}-\bar{Y}_{. .}\right)^{2} & =\sum_{i=1}^{a} \sum_{j=1}^{b_{i}}\left(Y_{i j}-\bar{Y}_{i .}\right)^{2}+\sum_{i=1}^{a} b_{i}\left(\bar{Y}_{i .}-\bar{Y}_{. .}\right)^{2} \\
& =Q_{1}+Q_{2}+\ldots+Q_{d} .
\end{aligned}
$$

Of particular interest is the fact that $\Delta_{1}=0$ if at least one $b_{i}>1$. The zero eigenvalue signifies that there is replication in the experiment (multiple observations per group) and thus an estimate for $\sigma_{2}^{2}$ is readily available. For the one-way random effects model, $Q_{1} \sim \sigma_{2}^{2} \chi^{2}\left(r_{1}\right)$ with $r_{1}=n-a$. In addition, $\sum_{i=1}^{a} \sum_{j=1}^{b_{i}}\left(Y_{i j}-\bar{Y}_{i .}\right)^{2}=Q_{1}$ and it follows that $\sum_{i=1}^{a} b_{i}\left(\bar{Y}_{i .}-\bar{Y}_{. .}\right)^{2}=Q_{2}+\ldots+Q_{d}$, which is distributed as a linear combination of scaled chi-squared variates. The analysis of variance table for any one-way random effects model of the form (1) is given in Table 1. 
In general, for a balanced one-way random effects model having $a$ groups and $b=n / a$ observations per group, $d=2$ with $\Delta_{1}=0, r_{1}=a(b-1), \Delta_{2}=b$, and $r_{2}=a-1$. For unbalanced designs having two distinct group sizes, there are at most three nonzero eigenvalues and closed-form solutions exist for $\Delta_{m}$ and $r_{m}$. For other unbalanced designs, numerical routines are needed to find $d, \Delta_{m}$, and $r_{m}$.

\section{Confidence Intervals for $\rho$}

\subsection{Exact Intervals for $\rho$}

For the general one-way random effects model given by (1), an exact confidence interval for $\rho$ may be obtained by recognizing that

$$
\frac{\sum_{m=2}^{d} \frac{Q_{m}}{1+\rho\left(\Delta_{m}-1\right)} / \sum_{m=2}^{d} r_{m}}{\frac{Q_{1}}{1-\rho} / r_{1}} \sim F\left(\sum_{m=2}^{d} r_{m}, r_{1}\right)
$$

where $F\left(d f_{1}, d f_{2}\right)$ denotes the $F$-distribution with $d f_{1}$ numerator degrees of freedom and $d f_{2}$ denominator degrees of freedom. See Burch and Iyer (1997) for alternative quantities which may used to construct confidence intervals for $\rho$. In general, the quantity in (6) is preferred since $Q_{2}, \ldots, Q_{d}$ are used to build the numerator and $Q_{1}$ is used to build the denominator. In other words, the quantity in (6) partitions $\left(Q_{1}, \ldots, Q_{d}\right)$ into $Q_{1}$ and $Q_{2}, \ldots, Q_{d}$, which is consistent with the division of the total variation into the "between groups" and "within groups" sources in Table 1.

Let $F_{\alpha / 2}$ and $F_{1-\alpha / 2}$ be the $\alpha / 2$ and $1-\alpha / 2$ percentiles of the $F$ distribution having numerator and denominator degrees of freedom equal to $\sum_{m=2}^{d} r_{i}=a-1$ and $r_{1}=n-a$, respectively. A $100(1-\alpha) \%$ confidence interval for $\rho$ is given by $(L(\mathbf{Q}), U(\mathbf{Q}))$ where

$$
P\left[F_{\alpha / 2} \leq \frac{\sum_{m=2}^{d} \frac{Q_{m}}{1+\rho\left(\Delta_{m}-1\right)} / \sum_{m=2}^{d} r_{m}}{\frac{Q_{1}}{1-\rho} / r_{1}} \leq F_{1-\alpha / 2}\right]=P[L(\mathbf{Q}) \leq \rho \leq U(\mathbf{Q})]
$$

and $\mathbf{Q}=\left(Q_{1}, \ldots, Q_{d}\right)$.

When $d=2$, as is the case for the balanced model (or unbalanced models with $a=2$ ) the endpoints of the confidence interval for $\rho$ are available in closed-form. They are

$$
\begin{aligned}
L(\mathbf{Q}) & =\frac{a(b-1) Q_{2}-(a-1) F_{1-\alpha / 2} Q_{1}}{a(b-1) Q_{2}+(a-1)(b-1) F_{1-\alpha / 2} Q_{1}} \\
U(\mathbf{Q}) & =\frac{a(b-1) Q_{2}-(a-1) F_{\alpha / 2} Q_{1}}{a(b-1) Q_{2}+(a-1)(b-1) F_{\alpha / 2} Q_{1}} .
\end{aligned}
$$

When $d>2$, however, the endpoints must be obtained via numerical methods. In either case, computing the expected length of the confidence interval, $E[U(\mathbf{Q})-L(\mathbf{Q})]$, can be 
cumbersome. Fortunately, a procedure given by Pratt (1961) can be used to accomplish this task.

Comparing the expected lengths of confidence intervals for $\rho$ from different one-way random effects designs will serve as a way to ascertain which designs yield the most precise inference about $\rho$. Designs which result in short intervals indicate more efficient use of the experimental material. Since expected length depends on the value of the parameter, one design may not be uniformly better than another design.

\subsection{Asymptotic Intervals for $\rho$}

Approximate confidence intervals for $\rho$ are based on the asymptotic distribution of the point estimator of $\rho$. The asymptotic properties of the ANOVA estimator of $\rho$ can be determined using regularity conditions. Burch and Harris (2001) show that

$$
\widehat{\rho} \stackrel{a s y m p}{\sim} N(\rho, V(\widehat{\rho}))
$$

where $V(\hat{\rho})$ is the asymptotic variance of $\hat{\rho}$ given by

$$
V(\widehat{\rho})=\frac{2(1-\rho)^{2}}{(n-a)(a-1)} \frac{\left(A \rho^{2}+B \rho+C\right)}{\bar{\Delta}^{2}}
$$

where

$$
\begin{aligned}
& A=(n-a) \operatorname{Var}\left(\Delta^{*}\right)+(n-1)(\bar{\Delta}-1)^{2} \\
& B=2(n-1)(\bar{\Delta}-1) \\
& C=n-1
\end{aligned}
$$

and $\bar{\Delta}=\sum_{m=2}^{d} r_{m} \Delta_{m} / \sum_{m=2}^{d} r_{m}, \operatorname{Var}\left(\Delta^{*}\right)=\sum_{m=2}^{d} r_{m}\left(\Delta_{m}-\bar{\Delta}\right)^{2} /(a-1)$. See Donna and Koval (1982) for further discussion of the ANOVA estimator of $\rho$. For a balanced design, the asymptotic variance of $\hat{\rho}$ reduces to

$$
V(\widehat{\rho})=\frac{2(n-1)(1-\rho)^{2}}{(n-a)(a-1)} \frac{(1+\rho(b-1))^{2}}{b^{2}} .
$$

This formula was first derived by Fisher (1925).

A $100(1-\alpha) \%$ asymptotic confidence interval for $\rho$ is given by $\widehat{\rho} \pm Z_{\alpha / 2} \sqrt{\hat{V}(\widehat{\rho})}$ where $\widehat{V}(\widehat{\rho})$ indicates that $\rho$ in (11) is replaced by $\hat{\rho}$ and $Z_{\alpha / 2}$ is associated with the $\alpha / 2$ percentile of the standard normal distribution. The expected length of the asymptotic confidence interval for $\rho$ is $2 Z_{\alpha / 2} \sqrt{V(\hat{\rho})}$. The asymptotic results rely on a normally distributed estimator whose distribution is not constrained to the unit interval. This may result in an interval whose endpoints fall outside the parameter space. While truncation issues are not addressed in the approximate method, they are dealt with in the exact method since the probabilities in (7) are unaffected by truncation. Truncation is particularly common when $\rho$ is small, so the asymptotic results may not be accurate for these cases. However, asymptotic intervals are easy to calculate and do provide guidance in finding optimal designs. 


\section{Examples}

The following examples illustrate the decision making process to implement the optimal allocation procedures described in this paper. In some cases a candidate design may not be uniformly optimal over the parameter space. The dependence on $\rho$ may be eliminated by computing the average expected length with respect to $\rho$ (a measure of the overall quality of the design), or the maximum expected length with respect to $\rho$ (a measure of the worst case scenario associated with the design).

\subsection{Fixed Sample Size: Find Best Balanced Design}

Suppose that the total sample size is fixed and that many balanced one-way random effects models are possible. The objective in this case is to find the best balanced design to estimate $\rho$. Returning to the apple orchard example, consider the case where $n=a b=100$. That is, 100 apples are to be picked with the condition that the same number of apples are picked from each tree. Table 2 lists the possible balanced designs for this scenario.

The seven designs in Table 2 are compared to one another in terms of the expected length of confidence intervals for $\rho$ using the exact calculations. Figure 1 displays the expected lengths of the $90 \%$ confidence intervals for $\rho$ where $\alpha / 2=0.05$. There does not exist a single design that has a uniformly minimum expected length across the parameter space. For example, if $\rho$ is greater than 0.65 , then Design 1 results in a confidence interval with minimum expected length. However, Design 1 does not fair as well as other designs if $\rho<0.65$.

Additional criteria may be used to determine the design of choice. For example, a minimax criterion would select the design with the minimum maximum expected length. A minimum average expected length criterion, where the averaging is over the possible parameter values, may yield a desirable design. For instance, one may select the design that minimizes

$$
\int_{0}^{1} E[U(\mathbf{Q})-L(\mathbf{Q})] d \rho .
$$

It is interesting to note that when the minimax or minimizing (16) criteria are employed, then Design 2, having 25 groups with 4 measurements per group, is the design of choice.

The expected lengths shown for the various designs in Figure 1 indicate that many groups with few measurements per group are desirable. In this example one would certainly not select, say, $b>5$. This leads to the possibility that depending on the sample size, optimal balanced one-way random effects models have $2 \leq b \leq 5$. To quantify this result, consider Figure 2. This figure displays that optimum choice of $b$ as a function of sample size for balanced designs using 90\% confidence intervals. Exact as well as asymptotic results are provided. Results are shown in terms of minimizing the maximum expected length. Similar results are obtained (although larger sample sizes are required) using the minimum 
average expected length. Using either criterion, as the sample size increases, the best $b$ increases from 2 to 3 to 4 and remains at 4 as the sample size goes to infinity.

Asymptotic confidence intervals for $\rho$ are computed using

$$
\begin{aligned}
E[U(\mathbf{Q})-L(\mathbf{Q})] & =2 Z_{\alpha / 2} \sqrt{V(\hat{\rho})} \\
& =2 Z_{\alpha / 2}\left(\sqrt{\frac{2(n-1)}{n(n-b)(b-1)}} \frac{1-\rho}{1+\rho(b-1)}\right) .
\end{aligned}
$$

This simple equation is due to the fact that $d=2, \operatorname{Var}\left(\Delta_{i}\right)=0$, and $\bar{\Delta}=b$ for any balanced one-way random effects model. As was the case using exact results, there does not exist a single design that has a uniformly minimum expected length across the parameter space. The average expected length of a confidence interval for a particular design using asymptotic theory is

$$
\begin{aligned}
\int_{0}^{1} E[U(\mathbf{Q})-L(\mathbf{Q})] d \rho & =2 Z_{\alpha / 2} \int_{0}^{1} \sqrt{V(\hat{\rho})} d \rho \\
& =2 Z_{\alpha / 2}\left(\frac{1}{6} \sqrt{\frac{2(n-1)}{n}} \frac{b+2}{\sqrt{n-b} \sqrt{b-1}}\right) .
\end{aligned}
$$

The asymptotic confidence interval comparisons yield tractable results as $\int_{0}^{1} \sqrt{V(\hat{\rho})} d \rho$ is minimized when

$$
b=\frac{2(2 n+1)}{n+5}
$$

If one considers the minimax approach, the maximum value of $\sqrt{V(\widehat{\rho})}$ is minimized when

$$
b=\frac{4 n}{n+3} .
$$

Using either criterion, the optimal design when there are several balanced designs to chose from is $b=4$ as the sample size goes to infinity. In other words, four observations per group provides more information about $\rho$ than does any other balanced design using asymptotic results.

\subsection{Fixed Sample Size: Find Best Design}

In this section we consider balanced as well as unbalanced designs. For a fixed value $n$, we seek the best overall design for estimating $\rho$. It is not always the case that the best design is a balanced design. Results using both asymptotic and exact calculations are presented.

For a fixed sample size, there does not exist one design that is uniformly better than another design since the asymptotic variance of $\hat{\rho}$ given by (11) depends on the value of 
$\rho$. Theoretical results based on the asymptotic approach are difficult to obtain. A search of all possible designs numerically (for a given $n$ ) reveals a consistent pattern. A heuristic way to state this rule is to select a design with as many groups of size 4 as possible, with the proviso that any remaining groups must be of size 3 . For example, $n=114$ could be distributed as 28 groups of size 4 and one group of size 2 , which would maximize the number of groups of size 4 , but this design violates the rule that remaining groups be of size 3. The unbalanced design of 27 groups of size 4 and two groups of size 3 outperforms the best balanced design, which is 38 groups of size 3 .

As was the case using the asymptotic approach, theoretical results based on exact calculations are not easy to produce. Whereas the asymptotic results indicate the optimal design has primary group sizes of four and secondary group sizes of three, exact calculations suggest smaller group sizes are needed for smaller sample sizes. In particular, designs having primary group sizes of 2 or 3 may be better for small sample sizes. However, these designs do not significantly outperform the design recommended using the asymptotic method.

Consider the example presented in Vangel (1992) with $n=25$ for which there are unbalanced designs that outperform the balanced design having $a=5$ and $b=5$. Figure 3 displays the expected lengths of the $90 \%$ confidence intervals for $\rho$ where $\alpha / 2=0.05$ for selected designs. The values of $b_{i}$ are listed next to each expected length curve. The designs having group sizes of 2 and 3, or 3 and 4, uniformly outperform the balanced design. The best design using the asymptotic minimax rule is $(3,3,3,4,4,4,4)$. The best design using the exact results is $(2,2,3,3,3,3,3,3,3)$. The percent relative difference of the maximum expected length for design $(3,3,3,4,4,4,4)$ compared to design $(2,2,3,3,3,3,3,3,3)$ is only $1.3 \%$.

\section{$5 \quad$ Summary and Conclusions}

We have presented rules for determining optimal designs based on expected length of confidence intervals for the intraclass correlation coefficient $\rho$. For simplicity, our recommendations are based primarily on theoretical results obtained from the asymptotic normal approximation to the ANOVA estimator of $\rho$. We have also performed extensive exact calculations of expected lengths for various sample sizes to bolster these conclusions.

If the total sample size is fixed, then optimal allocation of experimental units in a oneway random effects model depends on $\rho$. In the absence of knowledge of $\rho$, we suggest two methods; averaging of expected lengths over $\rho$ or considering the maximum expected length over $\rho$. Using asymptotic results, both methods suggest a group size of 4 . In general, $n$ will not be divisible by four, so a balanced design with groups of size four is not possible. In those cases we suggest selecting an unbalanced design having primary group sizes of 4 and secondary group sizes of 3 . 


\section{ACKNOWLEDGMENTS}

We would like to thank the referee for conducting a thorough review of the paper. A number of valuable suggestions were made that improved the quality of the paper.

\section{REFERENCES}

Burch, B. D., and Harris, I. R. (2002), "Optimal one-way random effects designs for the intraclass correlation based on confidence intervals," Technical Report SMU-TR-302, Southern Methodist University, Department of Statistical Science.

Burch, B. D., and Harris, I. R. (2001), "Closed-form approximations to the REML estimator of a variance ratio (or heritability) in a mixed linear model," Biometrics, 57, 1148-1156.

Burch, B. D., and Iyer, H. K. (1997), "Exact confidence intervals for a variance ratio (or heritability) in a mixed linear model," Biometrics, 53, 1318-1333.

Donner, A., and Koval, J. J. (1982), "Design considerations in the estimation of intraclass correlation," Annals of Human Genetics, 46, 271-277.

Fisher, R. A. (1925), Statistical Methods for Research Workers, Edinburgh: Oliver and Boyd.

LaMotte, L. R. (1976), "Invariant quadratic estimators in the random, one-way ANOVA model," Biometrics, 32, 793-804.

Pratt, J. W. (1961), "Length of confidence intervals," Journal of the American Statistical Association, 56, 549-567.

Vangel, M. G. (1992), "New methods for one-sided tolerance limits for a one-way balanced random-effects ANOVA model," Technometrics, 34, 176-185. 
Table 1: ANOVA Table for the One-way Random Effects Model

\begin{tabular}{lcc}
\hline Source & $\mathrm{df}$ & $\mathrm{SS}$ \\
\hline Between Groups & $a-1$ & $Q_{2}+\ldots+Q_{d}$ \\
Within Groups & $\sum_{i=1}^{a} b_{i}-a$ & $Q_{1}$ \\
\hline Total & $\sum_{i=1}^{a} b_{i}-1$ & $\sum_{i=1}^{a} \sum_{j=1}^{b_{i}}\left(Y_{i j}-\bar{Y}_{. .}\right)^{2}$
\end{tabular}

Table 2: Balanced Designs when $n=100$

\begin{tabular}{crr}
\hline Design & $\mathrm{a}$ & $\mathrm{b}$ \\
\hline 1 & 50 & 2 \\
2 & 25 & 4 \\
3 & 20 & 5 \\
4 & 10 & 10 \\
5 & 5 & 20 \\
6 & 4 & 25 \\
7 & 2 & 50 \\
\hline
\end{tabular}




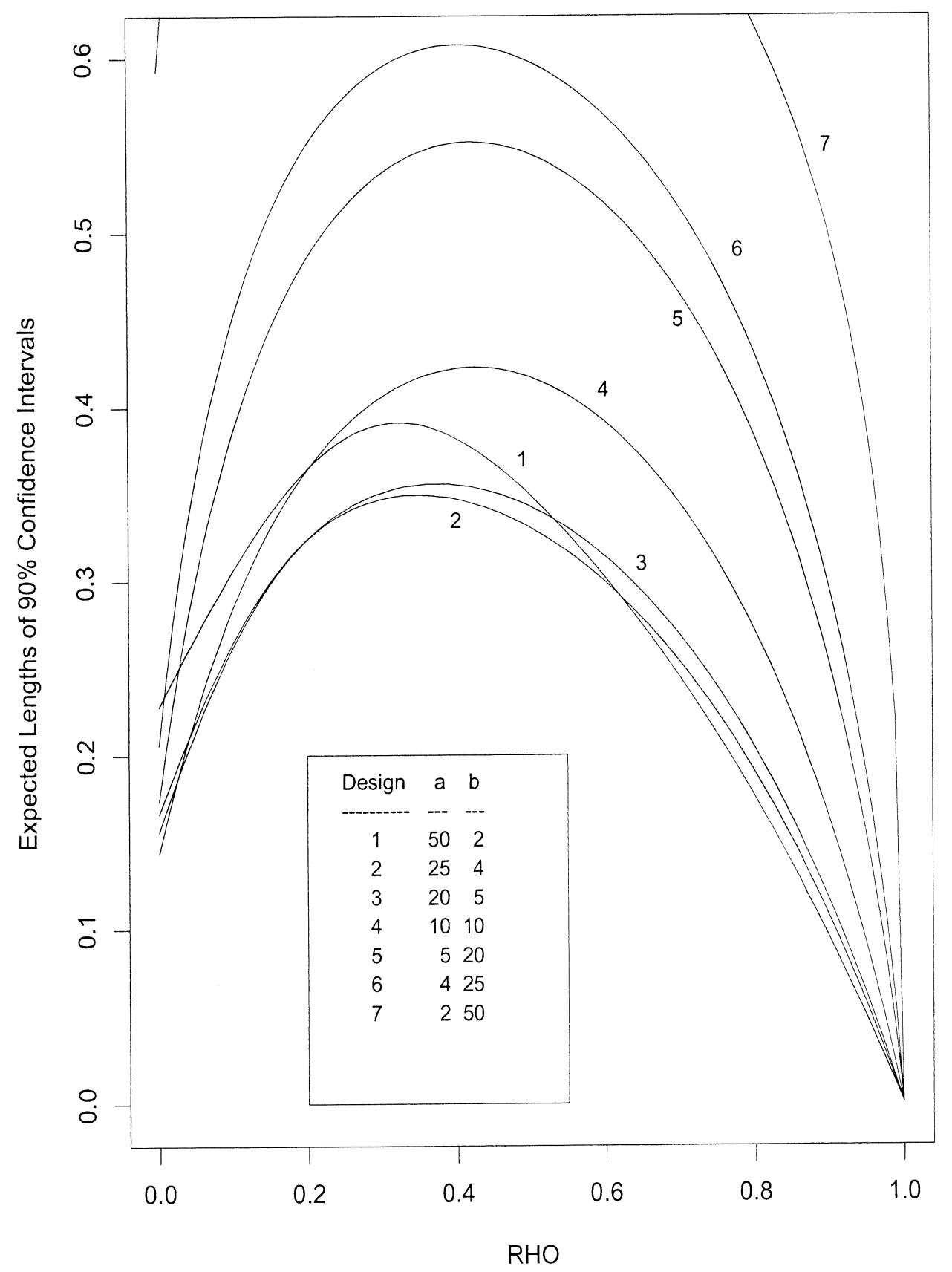

Figure 1: Comparing Balanced Designs when $n=100$ 


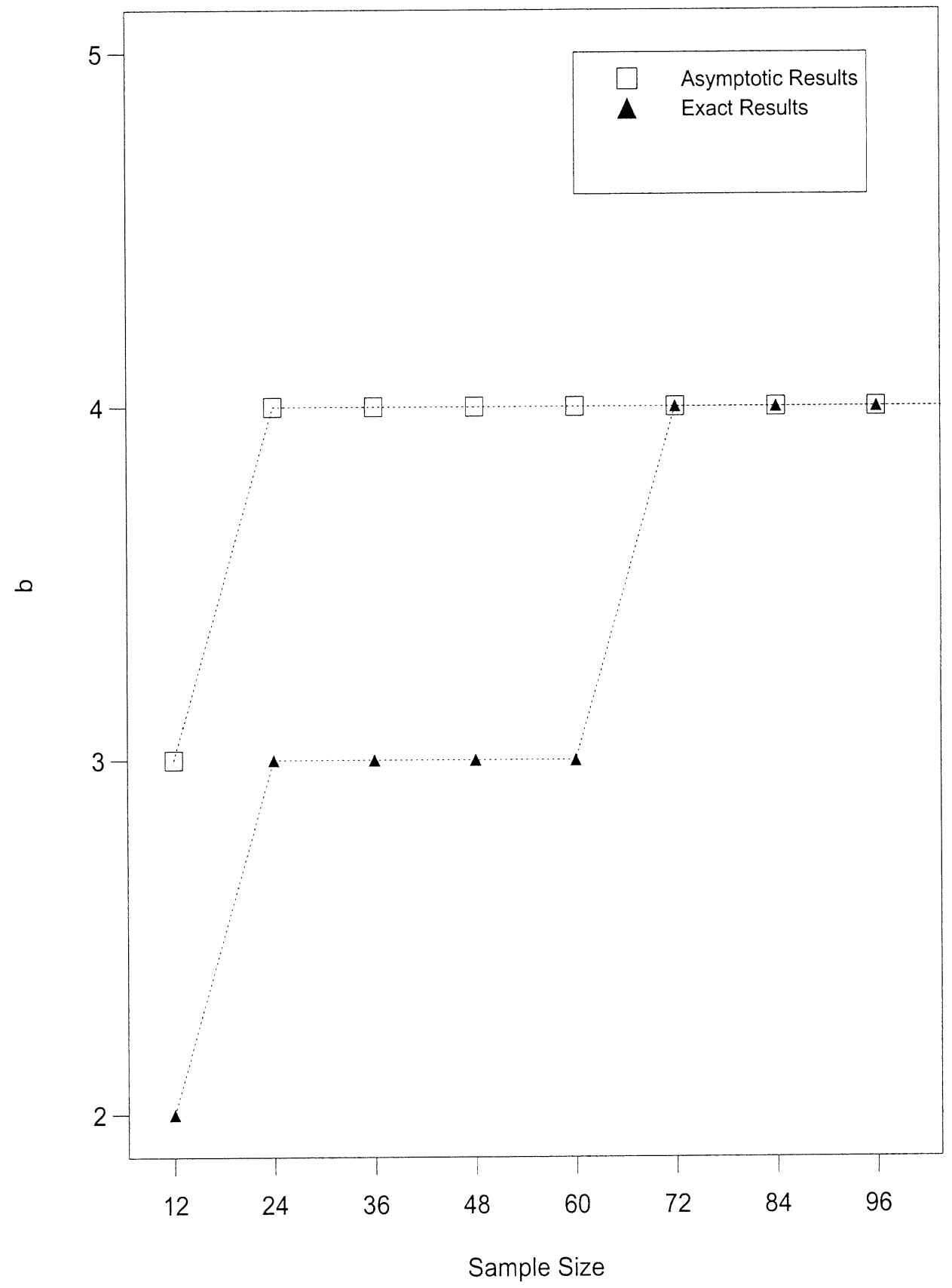

Figure 2: Optimal Group Sizes for Balanced Designs 


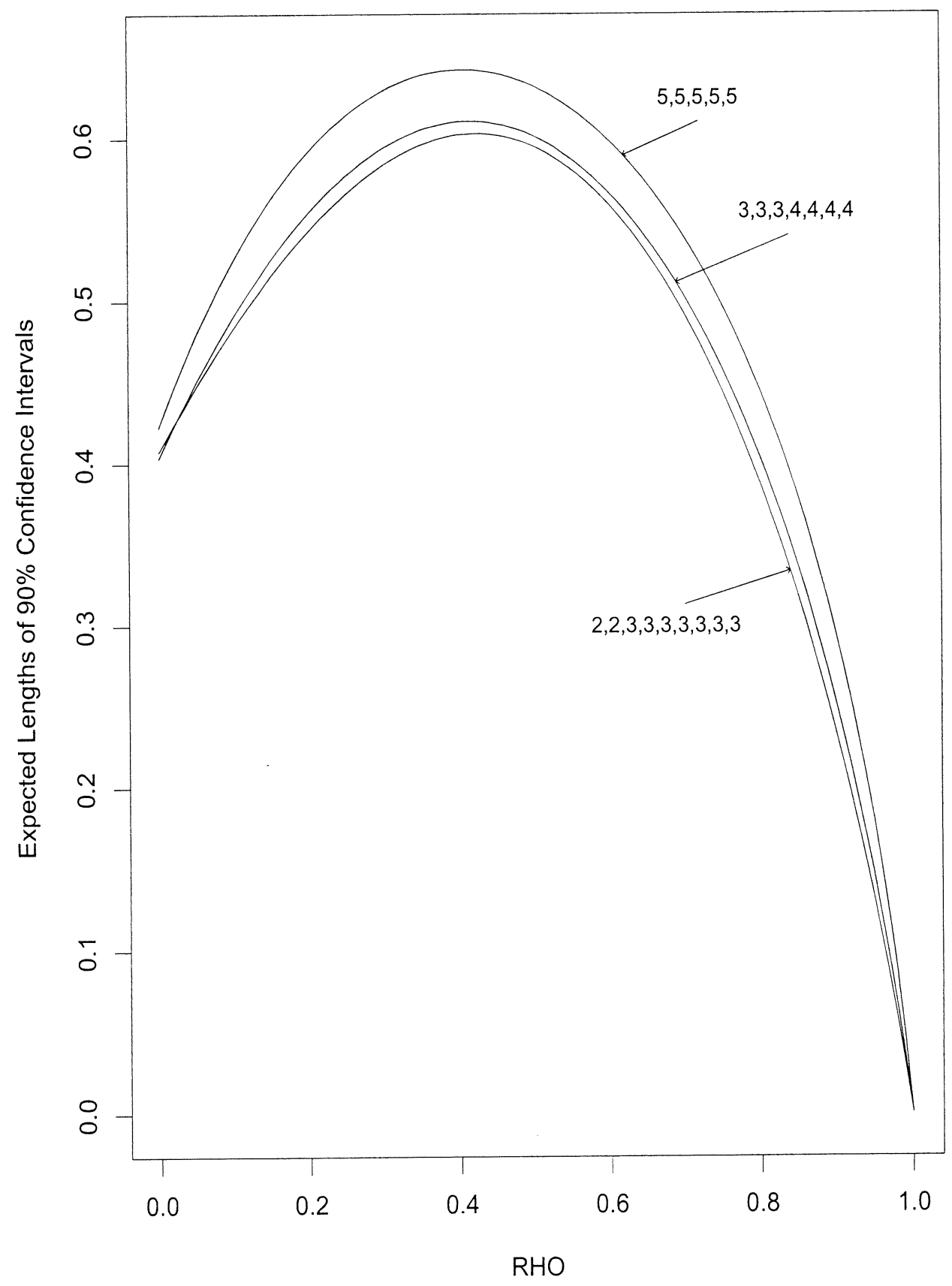

Figure 3: Comparing Balanced and Unbalanced Designs when $n=25$ 